\title{
Clinical Information Systems Integration in New York City's First Mobile Stroke Unit
}

\author{
Benjamin R. Kummer ${ }^{1,2,3}$ Michael P. Lerario $2,4,5$ Babak B. Navi ${ }^{2,4}$ Adam C. Ganzman ${ }^{4}$
}

Daniel Ribaudo ${ }^{6}$ Saad A. Mir ${ }^{2,4}$ Sammy Pishanidar ${ }^{2,4} \quad$ Tim Lekic $^{3}$ Olajide Williams ${ }^{3}$

Hooman Kamel ${ }^{2,4}$ Randolph S. Marshall ${ }^{3}$ George Hripcsak ${ }^{1}$ Mitchell S.V. Elkind ${ }^{3,7}$ Matthew E. Fink ${ }^{4}$

\footnotetext{
${ }^{1}$ Department of Biomedical Informatics, Columbia University, New York, United States

${ }^{2}$ Clinical and Translational Neuroscience Unit, Feil Family Brain and Mind Research Institute, Weill Cornell Medicine, New York, United States

${ }^{3}$ Department of Neurology, Columbia College of Physicians and Surgeons, New York, United States

${ }^{4}$ Department of Neurology, Weill Cornell Medicine, New York, United States

${ }^{5}$ Department of Neurology, New York-Presbyterian Queens, Flushing, New York, United States

${ }^{6}$ Department of Emergency Medical Services, New York Presbyterian Hospital, New York, United States

7 Department of Epidemiology, Mailman School of Public Health, Columbia University, New York, United States
}

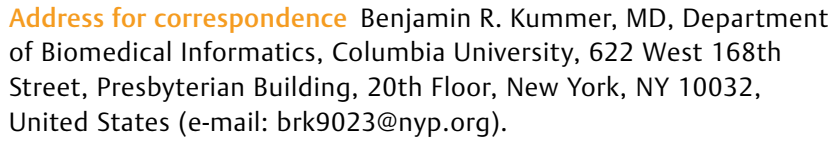

Address for correspondence Benjamin R. Kummer, MD, Department of Biomedical Informatics, Columbia University, 622 West 168th Street, Presbyterian Building, 20th Floor, New York, NY 10032, United States (e-mail: brk9023@nyp.org).

Appl Clin Inform 2018;9:89-98.

\section{Abstract}

Keywords

- stroke

- mobile health units

- integrated information systems

- implementation and deployment

- use, administration, and maintenance

- electronic health records and systems

- order entry

- radiology information systems

- clinical documentation and communications
Background Mobile stroke units (MSUs) reduce time to thrombolytic therapy in acute ischemic stroke. These units are widely used, but the clinical information systems underlying MSU operations are understudied.

Objective The first MSU on the East Coast of the United States was established at New York Presbyterian Hospital (NYP) in October 2016. We describe our program's 7-month pilot, focusing on the integration of our hospital's clinical information systems into our MSU to support patient care and research efforts.

Methods NYP's MSU was staffed by two paramedics, one radiology technologist, and a vascular neurologist. The unit was equipped with four laptop computers and networking infrastructure enabling all staff to access the hospital intranet and clinical applications during operating hours. A telephone-based registration procedure registered patients from the field into our admit/discharge/transfer system, which interfaced with the institutional electronic health record (EHR). We developed and implemented a computerized physician order entry set in our EHR with prefilled values to permit quick ordering of medications, imaging, and laboratory testing. We also developed and implemented a structured clinician note to facilitate care documentation and clinical data extraction.

Results Our MSU began operating on October 3, 2016. As of April 27, 2017, the MSU transported 49 patients, of whom 16 received tissue plasminogen activator (t-PA). Zero technical problems impacting patient care were reported around registration, order entry, or intranet access. Two onboard network failures occurred, resulting in received

July 25, 2017

accepted after revision

December 12, 2017
Copyright $\odot 2018$ Schattauer

DOI https://doi.org/

10.1055/s-0037-1621704

ISSN 1869-0327. 
computed tomography scanner malfunctions, although no patients became ineligible for time-sensitive treatment as a result. Thirteen (26.5\%) clinical notes contained at least one incomplete time field.

Conclusion The main technical challenges encountered during the integration of our hospital's clinical information systems into our MSU were onboard network failures and incomplete clinical documentation. Future studies are necessary to determine whether such integrative efforts improve MSU care quality, and which enhancements to information systems will optimize clinical care and research efforts.

\section{Background and Significance}

Acute stroke is a time-sensitive neurological emergency that represents a leading cause of death. ${ }^{1}$ In acute ischemic stroke, which is the most common type of acute stroke, thrombolytic treatment with tissue plasminogen activator (t-PA) as early as possible after symptom onset is associated with better chances of improved outcome. ${ }^{1,2}$ While many investigators have described systems-oriented approaches to reducing in-hospital time to thrombolysis, ${ }^{3,4}$ prehospital care delays remain a significant cause of prolonged treatment times, ${ }^{5}$ and efforts to decrease such delays are well-described. ${ }^{6,7}$ Mobile stroke units (MSUs), which are specialized ambulances equipped with onboard computed tomography (CT) and t-PA, are now operational in stroke centers internationally, and allow clinicians to deliver thrombolytic therapy for acute ischemic stroke in the prehospital setting faster than through traditional treatment systems in emergency departments. ${ }^{8-11}$ In October 2016, the first MSU in New York and the East Coast of the United States was established by New York Presbyterian (NYP) Hospital, in association with the Fire Department of New York. ${ }^{12}$

While many MSU programs currently exist across the world, the health information technology (IT) infrastructure employed by these programs has been understudied. In the United States, these infrastructures range from handwritten clinical documentation and order entry to full integration of the affiliated stroke center electronic health record (EHR)-including order entry and registration-into the MSU (Russman AA et al. New YorkPresbyterian Mobile Stroke Unit Introduction, personal communication, January 2017; Kummer BR, Jones W. Epic in the UC MSU, personal communication, June 2017; Lerario MP, Ganzman AC, Kummer BR, Harel A. Discussion of electronic health record usage in the NYP MSU program, personal communication, March 2017). While potentially more costly to implement in comparison to noncomputerized models, EHR and other forms of health IT such as electronic admit/discharge/transfer (ADT) or computerized physician order entry (CPOE) may allow for more accurate and interpretable clinical record-keeping, as well as more reliable and analyzable data to support operational, quality improvement-related, and research initiatives.

\section{Objective}

To our knowledge, no previous work has described the integration of an affiliated stroke center's clinical information systems into its MSU. Herein, we describe the integration of NYP's MSU into our hospital's clinical information systems to support patient care and research efforts during the program's initial 7-month pilot phase. In this descriptive study, we first describe the overview of the MSU program, followed by a description of its preimplementation phase, and basic workflows. We then present how each applicable workflow component was integrated into our hospital's clinical information systems during the development of our MSU program, and describe the implementation phase. We then include an account of ITrelated challenges. Since the objective of this study was to describe the integration of our hospital IT systems into the MSU, operational metrics-including transport times in the MSU and time to treatment with thrombolytic medication-were considered to be outside scope of this work, and were not included.

\section{Methods}

\section{Program Overview}

The MSU is recognized as an advanced cardiac life support unit by the emergency medical services network of New York, and is dispatched along with a basic life support ambulance unit to all suspected stroke alerts in the field. The crew is comprised of two paramedics, one radiology technologist, and one vascular neurologist. Currently, the MSU operates weekdays between 9 a.m. and 5 p.m., out of two medical campuses affiliated with NYP (Columbia University Medical Center and Weill Cornell Medical Center) in an alternating, biweekly schedule.

The MSU operates in two catchment areas (the Upper East Side and Washington Heights neighborhoods for Weill Cornell and Columbia University Medical Centers, respectively) depending on the biweekly operation period associated with each medical center campus. The Upper East Side population is $70 \%$ Caucasian, $23 \%$ foreign-born, and has a median household income of $\$ 132,493$ with $7 \%$ living under the national poverty level, ${ }^{13,14}$ whereas the Washington Heights communities comprise a $48 \%$ foreign-born population with a median household income of $\$ 37,460$, and more than $25 \%$ of households living below the national poverty level. ${ }^{15}$

\section{Preimplementation}

The preimplementation phase for the NYP MSU took place between January 2014 and October 2016. During this time, donor funds were secured, the vehicle housing the unit was ordered and built, and local stakeholders (including the Fire Department of New York and the Regional Emergency Medical Services Council of New York City) agreed to integrate the 
MSU into New York City's emergency dispatch system. Purchase, installation, and configuration of IT infrastructure occurred from June to September 2016, while clinical protocols were developed from July to August 2016, and workflow trainings for clinical personnel were held from August through September 2016.

Clinical and operational aspects of the NYP MSU program were largely based on the design and functioning of existing and successful MSU programs in Europe and the United States. Whereas publications of these programs' operational and clinical experience were reviewed by clinical leadership during preimplementation, no published accounts of MSU-related IT infrastructure implementation were available for review. Because the MSU was conceived as an extension of the hospital's emergency department, the MSU IT architecture was designed to match that of the hospital, including ADT, clinical documentation, and CPOE, as well as access to hospital intranet.

An MSU IT committee comprised of 12 individuals was also formed, and met weekly from July 2016 until October 2016. This committee consisted of two nursing-trained ADT specialists, who created dedicated MSU locations in the ADT system; two radiology technologists who installed the portable CT scanner, configured CT scanner software, and provided requirements for scanner connectivity over the MSU network; two network engineers who installed and configured the MSU's wireless and wired networks, including configuring access to the hospital intranet; two information services administrators who installed and configured the MSU's laptop computers; one emergency medical services administrator who purchased all hardware and software; one hospital neuroscience administrator, who aligned technical decisions with established service operations; and finally, two vascular neurologists, who both helped align technical decisions with clinical priorities, and served as unit testers.

To determine hours of operation, all available arrival time data from both medical center campuses was analyzed for patients with acute ischemic stroke presenting within 6 hours of symptom onset. A distribution of arrival time (not shown) according to 1-hour timeslots was compiled using data ranging from April 2008 to September 2016 at Columbia University Medical Center's campus $(N=1,252$ patients), and data from January 2016 to September 2016 $(N=76$ patients) at Weill Cornell Medical Center's campus.
This analysis demonstrated that most patients with acute stroke that were eligible for time-sensitive treatment arrived between the hours of 9 a.m. and 5 p.m.

Simulation exercises, which mimicked commonly encountered clinical scenarios in the MSU, were held weekly after installation and configuration of hardware and software. All crew members attended at least one exercise, and all simulation exercises were performed using the production environment and predetermined, fictitious patient records. During each exercise, patient registration and order entry were performed according to the requirements of the clinical scenario in each exercise.

\section{Clinical Workflow}

Prior to the launch of the MSU, the process of care for acute ischemic stroke followed a sequence of several events occurring in the field and in the hospital's emergency department ( - Fig. 1). In comparison to conventional care, the MSU allows administration of t-PA earlier by virtue of performing the neurological evaluation and CT scan in a specialized ambulance from the field, rather than performing these steps later in the emergency department.

The MSU clinical workflow is represented in - Fig. 2. After MSU dispatch and arrival at the scene of the emergency call, the MSU's vascular neurologist interviews and examines the patient, and decides whether the patient has symptoms consistent with an acute stroke. After MSU transport is deemed necessary, the patient is registered in the hospital ADT system via telephone and is brought into the MSU by paramedics. The clinician enters appropriate orders for the patient into the EHR; the patient is then scanned in a NeuroLogica CereTom (NeuroLogica Corporation, Danvers, Massachusetts, United States) portable CT scanner within the MSU's rear compartment. While the image is reviewed directly by the vascular neurologist in the MSU, an on-call neuroradiologist simultaneously reviews the study. If no contraindications to thrombolysis are present, the vascular neurologist then administers $\mathrm{t}$-PA in the MSU and the unit departs the scene. On arrival to the emergency department, care of the patient is transferred to a receiving emergency department physician and neurologist, whereupon blood samples are sent to the hospital laboratory. The clinician then documents the care encounter, as well as medication administration, in the EHR.
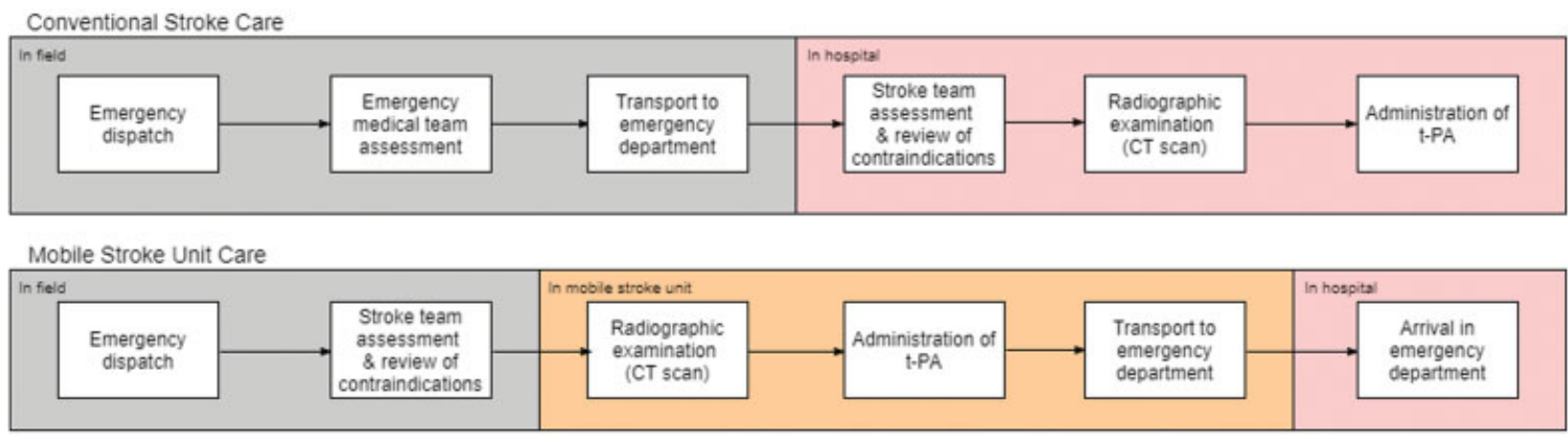

Fig. 1 Comparison of conventional (top) and mobile stroke unit (bottom) workflows, illustrating the earlier administration of tissue plasminogen activator (t-PA) in mobile stroke unit care. Processes in the gray sections occur in the field; processes in the orange sections occur within the mobile stroke unit; processes in the pink sections occur within the accepting hospital. 


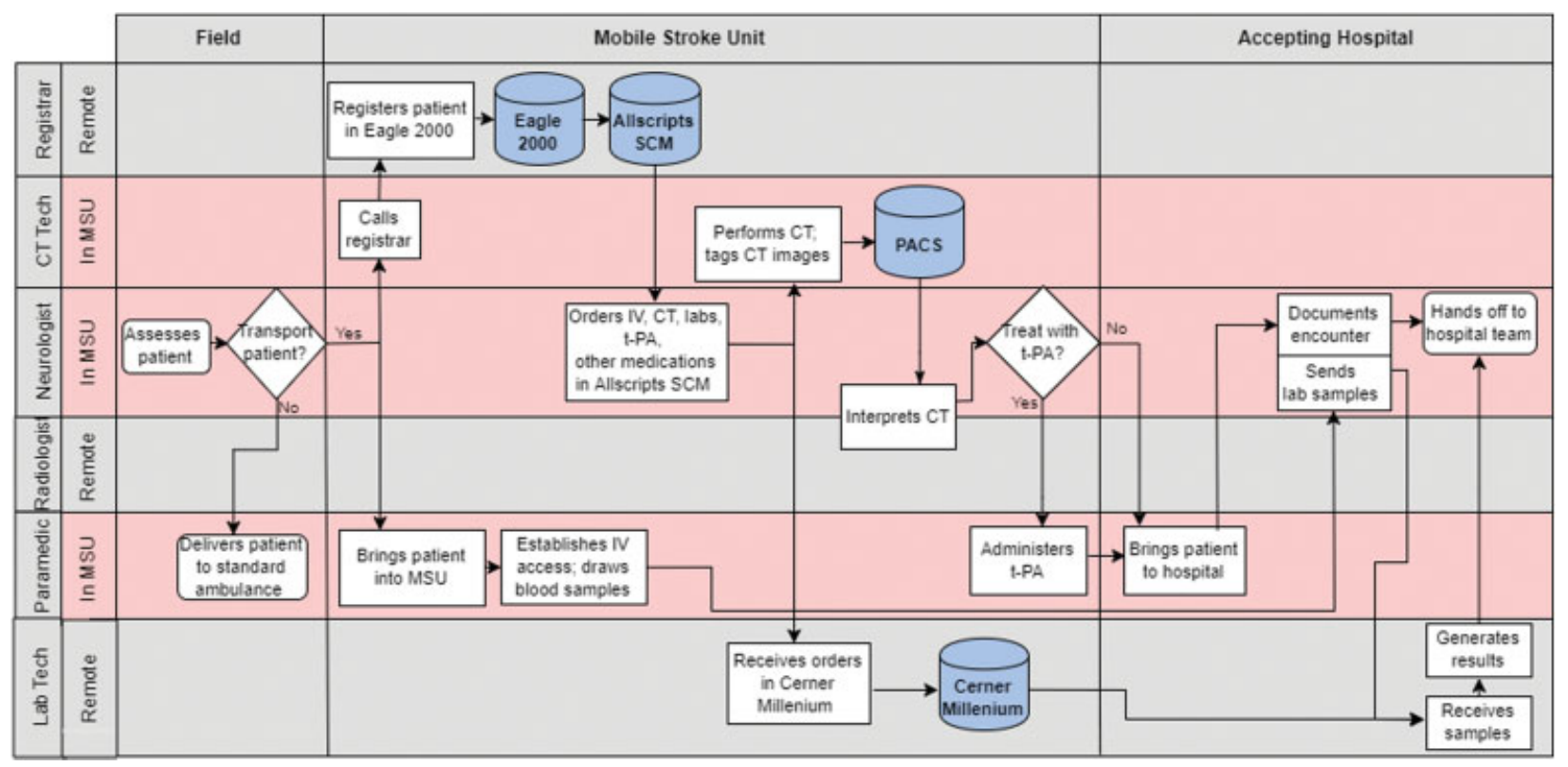

Fig. 2 Mobile stroke unit workflow. Care delivery locations are listed in top row; crew tasks are listed vertically in subsequent rows with respect to time (listed horizontally). Abbreviations: CT, computed tomography; IV, intravenous; MSU, mobile stroke unit; PACS, picture archive communication system; SCM, Sunrise Clinical Manager; tech; technologist; t-PA, tissue plasminogen activator.

\section{Clinical Applications}

Allscripts Sunrise Clinical Manager (SCM) (Allscripts Healthcare Solutions Inc., Chicago, Illinois, United States) is NYP's institutional EHR, and served as the principal application for clinical data review, documentation, and CPOE. Eagle 2000 (Cerner Corp., Kansas City, Missouri, United States), the NYP enterprise's ADT system, was used for patient registration; Eagle 2000 transmitted patient identification and visit information to SCM via an interface. NeuroLogica CereTom software, installed on a dedicated laptop in the MSU, enabled the acquisition of $\mathrm{CT}$ image data and subsequent transmission of this data via interface to a PACS (GE Centricity Web, GE Healthcare, Chicago, Illinois, United States). Centricity Imagecast (GE Healthcare), which was used to verify imaging studies and tag them with timestamps and other metadata, communicated with SCM via interface to receive clinicians' radiology study orders and transmit completed radiology reports (-Fig. 3). Each medical center campus hosted a separate instance of Imagecast, which was used during the designated 2-week MSU period belonging to each respective campus. Medical center radiologists at both campuses used Centricity RA1000 Workstation (GE Healthcare), which communicated with Imagecast via interface, to officially interpret the acquired images. Finally, Cerner Millennium (Cerner Corp.) served as the main application for processing laboratory orders and generating laboratory results. Similar to Eagle 2000 and Imagecast, this system communicated bidirectionally with SCM via interface, receiving clinician orders from SCM, and displaying laboratory results in the EHR for clinician review.

\section{Hardware}

One ruggedized, fourth-generation (4G)-based mobile access point (Cradlepoint Inc., Boise, Idaho, United States) was installed in the MSU and was used to set up a secure, dedicated network. A virtual private network tunnel was configured from the router to allow uninterrupted access to the hospital intranet and clinical applications. The unit was equipped with a total of four laptop computers; one laptop was used by the vascular neurologist for clinician order entry and documentation; a second machine was used by the radiology technician for tagging CT scan images with meta-data for each campus' instance of Imagecast. On any given 2-week period, the third, unused laptop served as the vascular neurologist's back-up machine. The fourth laptop computer was used by the radiology technician to control the $\mathrm{CT}$ scanner and acquire raw image data. The first three laptops were connected to the router via Ethernet cable whereas the dedicated CT laptop was connected wirelessly to the CT scanner via peer-to-peer network. Finally, the rear compartment of the MSU was equipped with two ruggedized Sprint cellular telephones to support patient registration, $\mathrm{CPOE}$, and communication between the MSU neurologist and the receiving inpatient neurology team.

\section{Patient Registration}

A dedicated MSU registration location was created in the ADT system. During the registration procedure, the $\mathrm{CT}$ technologist provided up to eight unique patient identifiers over telephone to an emergency department desk registrar, which triggered an automatic check for any preexisting records and selected the latter if present, in addition to generating a new visit record (or a new medical record number if no preexisting record was present). Both medical record and visit numbers were then communicated to SCM via interface, and were used in SCM to enter clinical orders and documentation.

\section{Clinician Order Entry}

To facilitate quick ordering of medications, imaging, and laboratory testing in the MSU setting that would be necessary to 


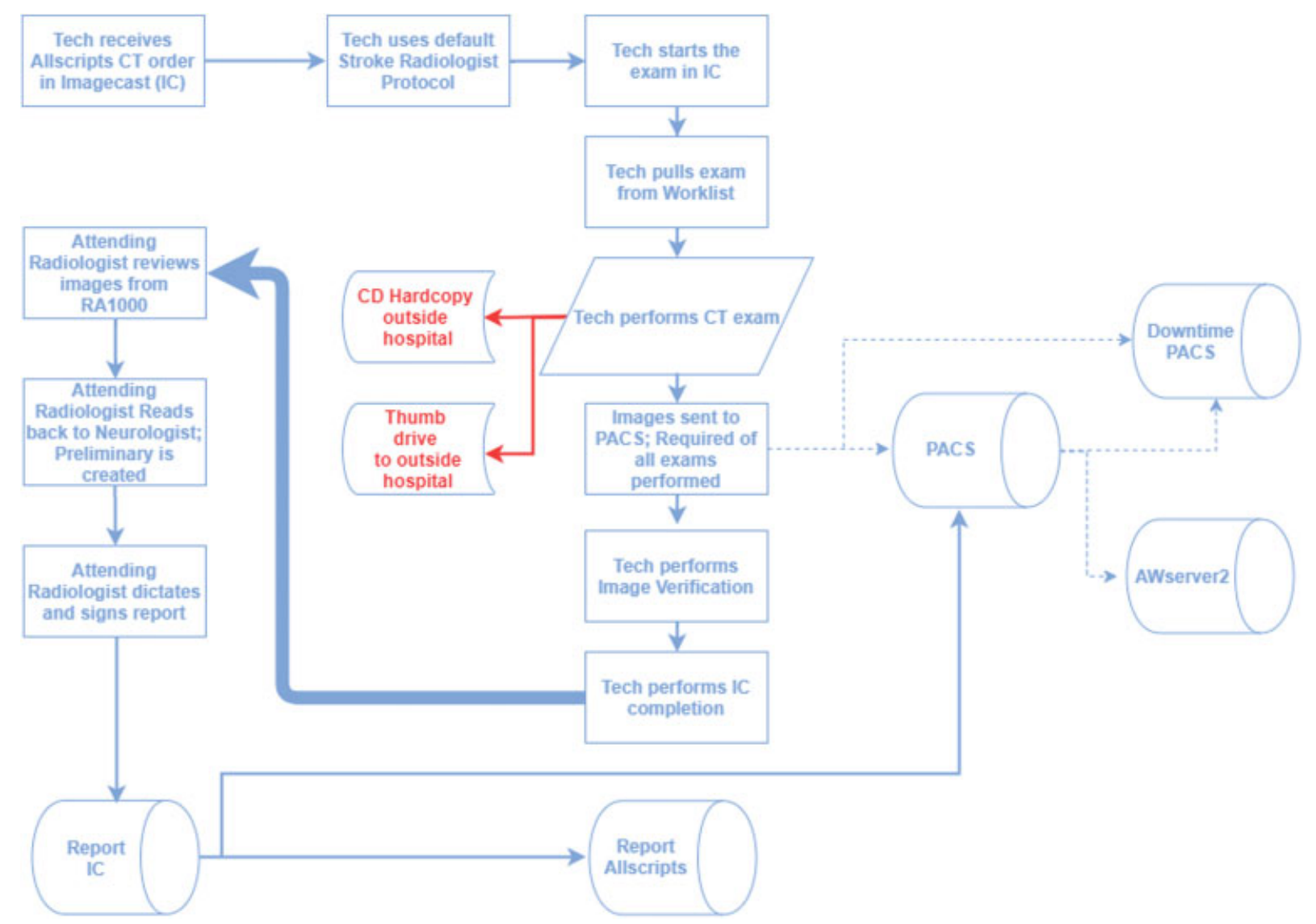

Fig. 3 Mobile stroke unit radiology process flow. Abbreviations: AWserver2, Advantage Workstation Server generation 2; CD, compact disc; CT, computed tomography; PACS; picture archive communication system; RA1000, GE Centricity RA1000 Workstation; Tech, technologist; WO, without.

support time-sensitive stroke care, a dedicated CPOE set (- Fig. 4) was built and implemented in SCM based on input from the departments of neurology, laboratory services, and radiology services. After patient registration, the clinician opened the standard clinical order entry window in SCM and selected the CPOE set, which was named "Mobile Stroke Unit Order Set" and consisted of a list of basic nursing orders (two intravenous lines, finger-stick glucose), and orders for three intravenous medications (labetalol, nicardipine, and alteplase), laboratory tests, and noncontrast CT scan of the head. While the clinician had to select orders by checkbox, all orders were by default set to clinically appropriate values, "STAT" priority, and the MSU's onboard cell phone number as contact information.

One stroke nurse practitioner (A.C.G.) drafted a specification document of this CPOE order set, and obtained approval by two required hospital IT governance committees in August 2016. The first committee (Health Information Management) reviewed justification of the business case for the order set, whereas the second committee (Order Set Committee) reviewed the specification for usability and consistency with existing CPOE sets in Allscripts SCM. The order set was then built by a software engineer and was unit-tested by two vascular neurologists (B.R.K., M.P.L.) and the same stroke nurse practitioner before being implemented to the production environment in late September 2016.

\section{Radiology}

A detailed process for the acquisition, transmission, and recording of radiology imaging data ( - Fig. 3) was created based on input from the departments of neurology, emergency medical services, and radiology services. Immediately after image acquisition in the MSU, the technologist reviewed the scan for technical errors and tagged the images with timestamp metadata, and the image was routed to PACS. The completed radiology study was then routed over the secure intranet connection to the RA1000 client, where it was reviewed by an on-call neuroradiologist and preliminarily interpreted before being transmitted to Imagecast via interface.

\section{Laboratory Testing}

To avoid repeat postthrombolysis venipuncture, blood samples were drawn on the MSU by paramedics. At the time the patient was brought into the MSU, laboratory orders were entered into SCM through the CPOE set and transmitted via interface to Cerner Millennium. After arrival to the emergency department and delivery of samples drawn on the MSU to the laboratory facility, samples were run and results generated by Cerner Millennium, which were then transmitted back to SCM and displayed there for clinician review. 


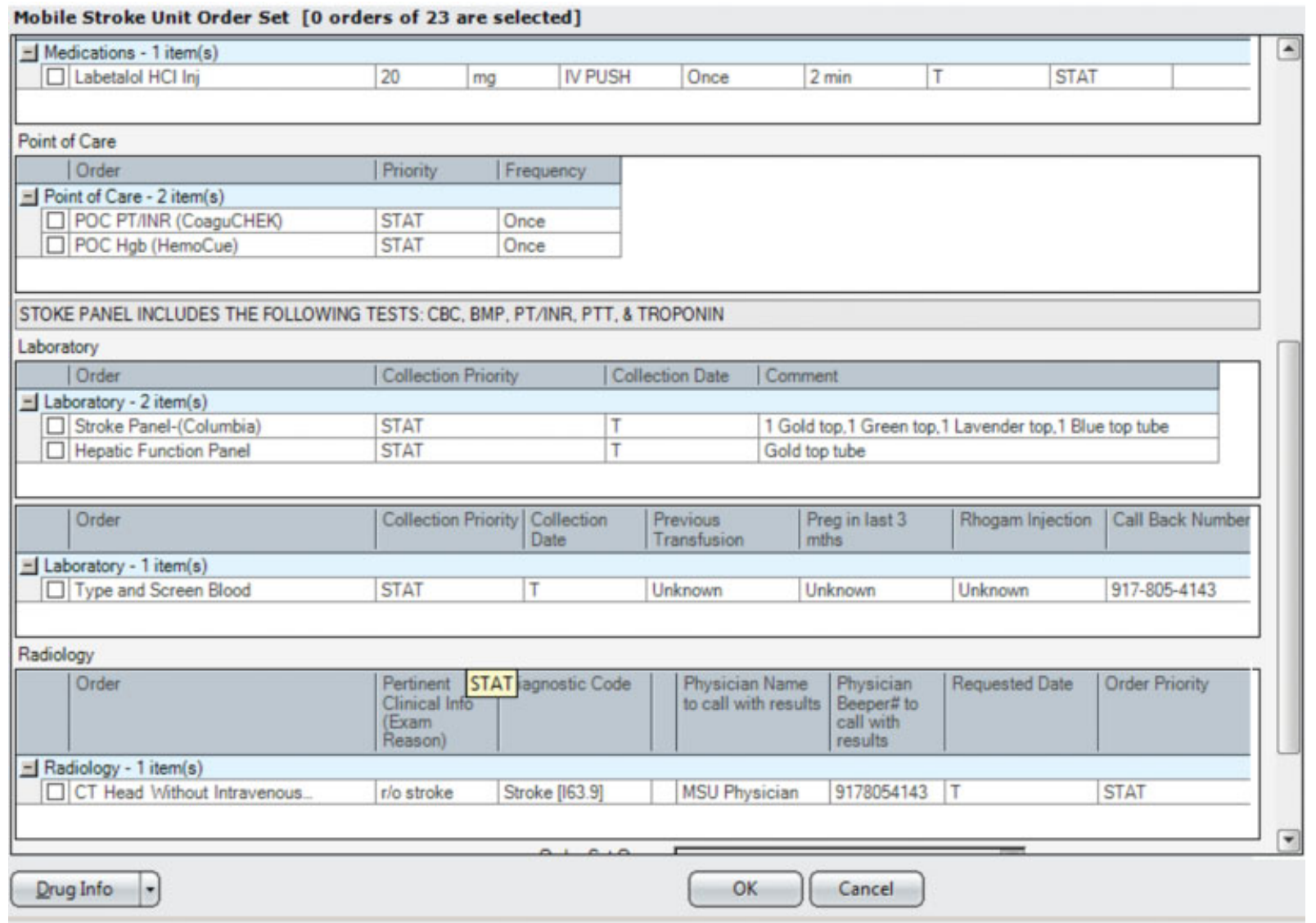

Fig. 4 Mobile stroke unit computerized physician order entry (CPOE) window snapshot. Pictured are one of three intravenous medications, laboratory tests, and radiology orders (nicardipine, tissue plasminogen activator, and nursing orders not pictured). Abbreviations: BMP, basic metabolic panel; CBC, complete blood count; CT, computed tomography; Hgb, hemoglobin; INR, international normalization ratio; IV, intravenous; PT, prothrombin time; PTT, partial thromboplastin time; r/o, rule out.

\section{Clinical Documentation}

A clinical note ( - Fig. 5) was created and implemented in SCM to document the physician encounter in the MSU based on input from key neurology department users, as well as hospital clinical documentation specialists. From July 2016 to August 2016, one vascular neurologist (B.R.K.) gathered requirements for the note from clinical leadership, drafted a specification document, and obtained approval from required hospital IT governance committees to proceed with development. To facilitate data retrieval for quality analysis and research efforts, the note was specifically designed to capture pertinent clinical details (including past medical history, medications, and stroke care timestamps) in structured fields wherever possible ( - Table 1). Past medical history and medications sections contained a combination of free-text boxes and checkbox widgets, with the latter associated with commonly encountered values, such as "hypertension" or "hyperlipidemia" for medical history, and "aspirin" or "warfarin" for medications; the free-text option was included to accommodate "not applicable" cases. As shown in - Table 1, a minority of note fields contained unstructured text data. No fields were required to save the note.

After approval from IT governance, two software engineers were then recruited to build the clinical note in September 2016. Throughout the same month, the same vascular neurologist performed several rounds of unit-testing of the note prototypes in the development environment. Once unit-testing was complete, the note was then implemented in the production environment in early October 2016.

\section{Implementation}

The program was launched on October 3, 2016 and consisted of regular 8-hour shifts during the aforementioned hours and days of operation without any preemptive limitation of operating times. At the end of each clinical shift for the first 2 weeks of operation, daily debriefing conferences were held between members of the IT and clinical steering committees and the crew riding in the MSU on that given day's shift. During this time, all clinical cases transported on the MSU were reviewed with all attendees, and both committees were asked to discuss any IT challenges or malfunctions that arose. After approximately 2 weeks, the operations of the MSU became routine, such that daily debriefings were no longer necessary. Each case transported on the MSU was logged in a dedicated MSU, and data from the clinical encounter note was extracted manually for each case. The pilot phase of this implementation occurred from October 2016 to April 2017.

The implementation of the IT infrastructure in the MSU only affected the conventional care workflow through slight modifications to Fire Department dispatch protocols, which 


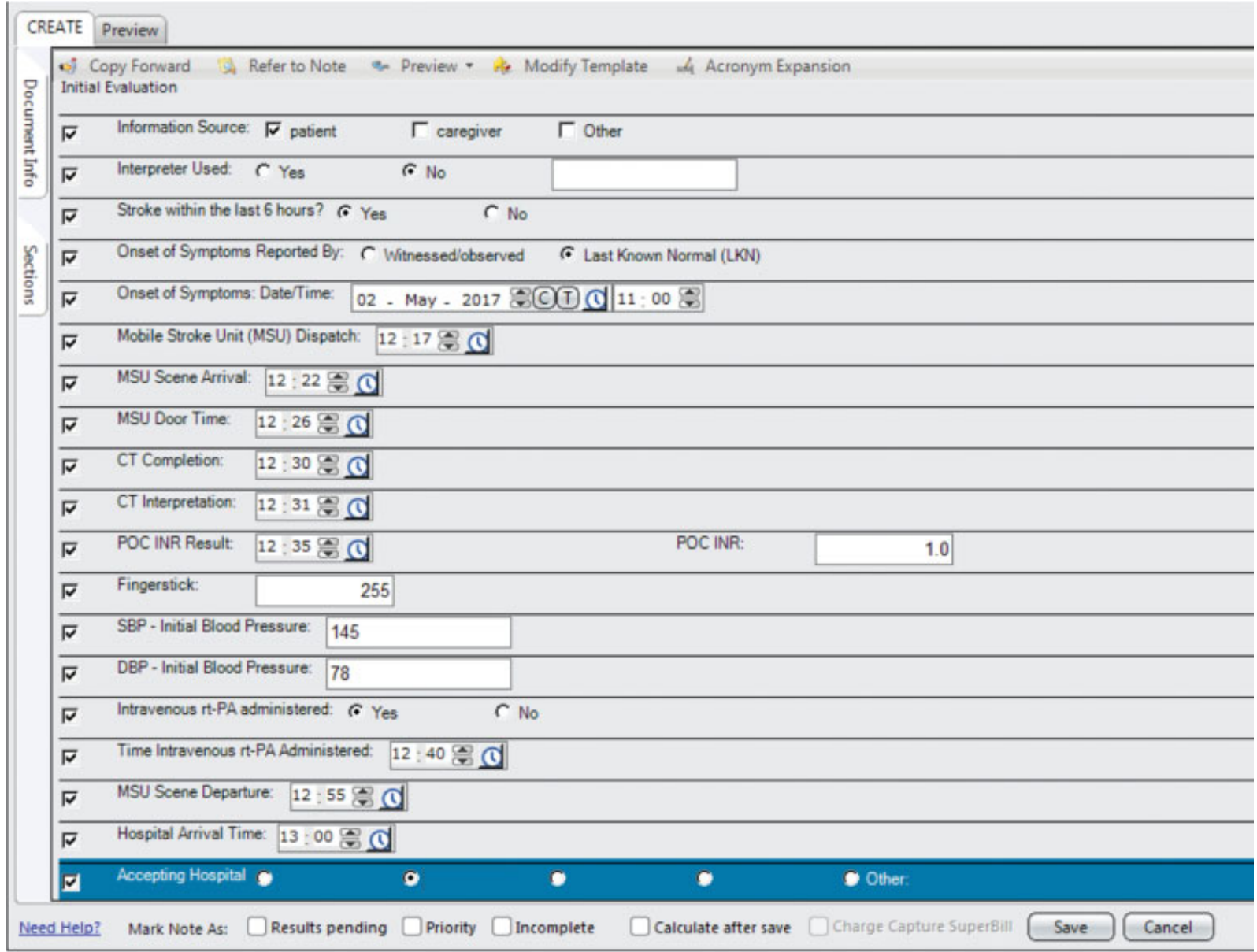

Fig. 5 Mobile stroke unit clinical note snapshot. Pictured is the note's topmost section. Note: Past medical history, medications (including antiplatelet and anticoagulant use), social history, family history, physical examination, assessment, and plan sections not shown. Identifying radio button options have been covered to preserve blinding. Abbreviations: CT, computed tomography; INR, international normalized ratio; POC, point of care; rt-PA, recombinant tissue plasminogen activator.

needed to be made to accommodate the MSU in their preexisting citywide stroke response algorithms. Particularly, during 2-week periods when the MSU was not available at one of the two NYP medical center campuses, there was no modification to the pre-MSU care workflow for transporting and treating patients with acute stroke.

\section{Results}

As of April 27, 2017, the NYP MSU transported 49 patients, of whom $49 \%$ had acute ischemic stroke, $41 \%$ had a final diagnosis of nonstroke, $6 \%$ had intracerebral hemorrhage, and $3 \%$ had transient ischemic attack (-Table 2). Sixteen (32.6\%) patients were treated with intravenous t-PA. The MSU's operational time metrics, including times to treatment with t-PA and safety outcomes, will be published in detail separately. Twenty-five neurologists participated in taking clinical shifts on the MSU, of whom 13 were vascular neurology-trained and 12 were neurocritical care-trained. Zero problems involving connectivity to the hospital intranet, clinical documentation, or CPOE occurred that directly impacted patient care (-Table 3 ). However, two significant CT malfunctions occurred. On the first occasion, network connectivity was lost between the ImageCast laptop and scanner; on the second occasion, peer-to-peer network connectivity was lost between the dedicated Ceretom laptop itself and the scanner. In both cases, the delays caused by CT malfunctions necessitated the patient's being routed to the nearest stroke center for CT scanning prior to thrombolysis, but did not prevent treatment with t-PA.

On the first occasion, an equipment check of the MSU rear compartment was performed as a first response immediately after patient care was rendered; a broken Ethernet cable was found between the Imagecast laptop and the router, and the CT was restored to normal functioning following replacement of the Ethernet cable. An equipment-checking protocol was also implemented for the CT technologist at the beginning of each MSU shift to minimize usage of broken equipment. On the second occasion, network diagnostics on the unit's peerto-peer network revealed a conflict between the Ceretom scanner's operating system and the network, which caused a lapse in network connectivity between the scanner and the dedicated CT laptop. The NeuroLogica vendor shortly thereafter installed a wireless access point on the scanner, enabling both devices to communicate via a second wireless local-area (as opposed to a peer-to-peer) network connection and resulting in 
Table 1 Mobile stroke unit clinical note fields

\begin{tabular}{|l|l|l|}
\hline Time-related & Data type & Note widget \\
\hline Date of service & Date & Date chooser \\
\hline Last known well time & Time & Time chooser \\
\hline Dispatch time & Time & Time chooser \\
\hline Scene arrival time & Time & Time chooser \\
\hline CT head completion time & Time & Time chooser \\
\hline CT head interpretation time & Time & Time chooser \\
\hline Point-of-care INR time & Time & Time chooser \\
\hline Scene departure time & Time & Time chooser \\
\hline t-PA administration time & Time & Time chooser \\
\hline Hospital arrival time & Time & Time chooser \\
\hline Nontime-related & & \\
\hline Interpreter use & Boolean & Radio button \\
\hline Source of history & Varchar & Radio button \\
\hline Finger stick glucose value & Integer & Text box \\
\hline Point-of-care INR value & Decimal & Text box \\
\hline Accepting hospital & Varchar & Radio button ${ }^{\text {a }}$ \\
\hline Medical history & Varchar & Checkbox \\
\hline $\begin{array}{l}\text { Antiplatelet agents } \\
\text { on admission }\end{array}$ & Varchar & Checkbox \\
\hline onticoagulant agents & Varchar & Checkbox \\
\hline History & Varchar & Free-text \\
\hline Physical examination & Varchar & Free-text \\
\hline Assessment & Varchar & Free-text \\
\hline Plan & Varchar & Free-text \\
\hline Abron
\end{tabular}

Abbreviations: CT, computed tomography; INR, international normalized ratio; t-PA, tissue plasminogen activator; Varchar, character string of variable character length.

a Featured a free-text option to accommodate "not applicable" cases.

no further network drop-offs. As of April 27, 2017, 13 (26.5\%) notes were missing at least one structured time field. All notes were completed after MSU patients had been delivered to a receiving hospital.

\section{Discussion}

We describe the integration of our institution's clinical information systems into our MSU to support clinical care and research efforts. This endeavor involved closely collaborating between clinical and nonclinical departments across the NYP hospital system and two separate medical center campuses, as well as coordinating multiple clinical data flows, including registration, radiology, laboratory, clinician documentation, and CPOE. We also show that while minimal interruptions in service occurred due to information systems during the first 6 months of our unit's operation, CT malfunctions impacting patient care did occur due to
Table 2 Characteristics of patients transported on the MSU $(N=49)$

\begin{tabular}{|l|l|}
\hline Characteristic $^{\text {a }}$ & \\
\hline Age in years, mean (SD) & $75.8(18.1)^{\mathrm{b}}$ \\
\hline Female & $26(53.1)$ \\
\hline Received intravenous t-PA & $16(32.6)$ \\
\hline Final diagnosis & \\
\hline Acute ischemic stroke & $24(49.0)$ \\
\hline Nonstroke & $20(40.8)$ \\
\hline Intracerebral hemorrhage & $3(6.1)$ \\
\hline Transient ischemic attack & $2(4.1)$ \\
\hline
\end{tabular}

Abbreviations: MSU, mobile stroke unit; SD, standard deviation; $\mathrm{t}-\mathrm{PA}$, tissue plasminogen activator.

${ }^{a}$ Data are presented as number (\%) unless otherwise specified.

${ }^{\mathrm{b}} \mathrm{N}=48$, as one patient was transferred to an outside hospital emergently and age was not recorded.

Table 3 Number and description of IT-related problems encountered during MSU implementation

\begin{tabular}{|l|l|l|}
\hline Category & Number & Description \\
\hline $\begin{array}{l}\text { Clinical } \\
\text { documentation }\end{array}$ & $13(26.5)$ & $\begin{array}{l}\text { Incomplete } \\
\text { documentation }\end{array}$ \\
\hline CT scanning & $2(4.1)$ & $\begin{array}{l}\text { Broken patch cable } \\
\text { Failure of peer-to-peer } \\
\text { network connection }\end{array}$ \\
\hline CPOE & 0 & NA \\
\hline $\begin{array}{l}\text { Intranet } \\
\text { connectivity }\end{array}$ & 0 & NA \\
\hline Registration & 0 & NA \\
\hline
\end{tabular}

Abbreviations: $\mathrm{CPOE}$, computerized physician order entry; $\mathrm{CT}$, computerized tomography; IT, information technology; MSU, mobile stroke unit; NA, not applicable.

Note: Only problems affecting patient care were reported. $N=49$ patients for all calculations. Data are presented as number (\%) unless otherwise specified.

hardware and software failures, without impairing eligibility for time-sensitive stroke treatment. We additionally show that nearly a quarter of clinician encounter notes were missing at least one time field.

While physician fatigue and technical difficulties (e.g., as in the case of both CT malfunctions, where CT completion and interpretation times had to be left blank) are plausible causes, incomplete documentation is most likely attributable to the lack of required fields in the MSU clinical encounter note. This note was originally designed without required fields to prevent the clinician from being distracted by interruptive alerts while delivering time-sensitive care for a patient with stroke inside the MSU. While this strategy was theoretically advantageous from a patient safety perspective, in the first 6 months of operation, all MSU clinical notes were completed after patient's arrival to receiving hospitals due to the constant attention required during clinical care and the short travel time between pick-up location and receiving 
hospitals. Given that several stroke care quality and research metrics are based on time data (such as time from symptom onset to t-PA treatment, as well as time from team arrival to CT scan), ${ }^{2,16}$ the effect of this missing time data on measuring care quality and research is nonnegligible. Potential solutions to such a problem include educational campaigns for MSU clinicians or interruptive alerts to force clinicians to complete important data fields. As opposed to the latter solution, which has the distinct disadvantage of workflow interruption, a dictation system with automatic voice recognition software could present a third and potentially less intrusive solution that could help accelerate clinical information (such as timestamps or medical history) transfer into the EHR and improve data accuracy.

Our study benefits from several strengths. First, this is the first report, to our knowledge, of the integration of a hospital's clinical information systems into an MSU. Second, we include a detailed rendition of our MSU's information systems architecture, which can be used as a template for other programs, and may be especially useful considering the expansion of MSU programs across the world. Third, our experience underlines the importance of multidisciplinary input in the development of our MSU information systems architecture. While these strengths are important, our study is limited by several factors, the first of which is the descriptive nature of our study. Second, because our MSU program's information systems were integrated into our hospital's existing systems from inception, it is not possible to examine the effect of integration on care quality through a comparison of pre- and postintegration states. Third, our architecture's reliance on the Allscripts EHR may not be generalizable to other institutions, which may use a different core EHR for their clinical operations. Finally, we do not include data on the cost of our integration efforts.

In summary, the architecture of the NYP MSU, which featured a full integration of the MSU into NYP's existing clinical information systems, was the cornerstone for prehospital stroke care delivery at our institution. Benefits of system integration were generation of clear clinical documentation and structured MSU data, which could then be extracted and analyzed for the purposes of quality improvement and research in prehospital stroke care. However, our experience did involve a lengthy planning process, engagement of multiple stakeholders, investment in technical infrastructure, and customized health IT solutions such as clinician documentation and CPOE sets. Additionally, software and hardware malfunctions did impact clinical care in two cases, and incomplete clinician documentation posed a challenge to data quality.

As MSUs become increasingly widespread, data supporting their use will likely become increasingly necessary. Moreover, as health systems worldwide increasingly use EHRs and other forms of health IT to support clinical activities, conducting information system integration such as the one we describe will become ever more important. Further studies are nonetheless needed to better understand the comparative benefits of MSUs using integrated clinical information systems over handwritten models of clinical workflow and data collection. Additionally, as our MSU program and others of its kind continue to operate and expand, it will be important to conduct additional investigations on which types of enhancements in clinical information systems will optimize prehospital stroke care quality.

\section{Clinical Relevance Statement}

Clinical information systems integration was feasible in a MSU associated with New York Presbyterian Hospital, although significant tradeoffs were apparent between data quality and ease-of-use, as well as data quality and investment in infrastructure. It is currently unknown whether integrated clinical information systems in a MSU are better for care quality than handwritten models, and which clinical information systems can optimize MSU care quality.

\section{Multiple Choice Question}

Which information process presented major challenges in the integration of the mobile stroke unit into the hospital's existing clinical information systems?
a. Admit/discharge/transfer (ADT) system registration
b. Computerized physician order entry (CPOE)
c. Clinical encounter documentation
d. Connectivity to hospital intranet

Correct Answer: The correct anwer is c, clinical encounter documentation. In the experience of NYP's MSU systems integration, out of all other options, only clinical documentation presented major challenges to the implementation, given that up to $26 \%$ of all notes contained at least one incomplete time field. The other main area of implementation challenge was CT scanning, but this option is not listed in the options above.

Protection of Human and Animal Subjects

The institutional review boards of Columbia University and Weill Cornell Medical Centers both approved this study.

Conflict of Interest

None.

Acknowledgment

The authors would like to thank Ariella Harel, Emil Smith, and Michael Koppel for their guidance and leadership during the development and launch of the NYP MSU program.

\section{References}

1 Benjamin EJ, Blaha MJ, Chiuve SE, et al; American Heart Association Statistics Committee and Stroke Statistics Subcommittee. Heart disease and stroke statistics-2017 update: a report from the American Heart Association. Circulation 2017;135 (10):e146-e603

2 Jauch EC, Saver JL, Adams HP Jr, et al; American Heart Association Stroke Council; Council on Cardiovascular Nursing; Council on Peripheral Vascular Disease; Council on Clinical Cardiology. 
Guidelines for the early management of patients with acute ischemic stroke: a guideline for healthcare professionals from the American Heart Association/American Stroke Association. Stroke 2013;44(03):870-947

3 Ruff IM, Ali SF, Goldstein JN, et al. Improving door-to-needle times: a single center validation of the target stroke hypothesis. Stroke 2014;45(02):504-508

4 Meretoja A, Strbian D, Mustanoja S, Tatlisumak T, Lindsberg PJ, Kaste M. Reducing in-hospital delay to 20 minutes in stroke thrombolysis. Neurology 2012;79(04):306-313

5 Evenson KR, Foraker RE, Morris DL, Rosamond WD. A comprehensive review of prehospital and in-hospital delay times in acute stroke care. Int J Stroke 2009;4(03):187-199

6 Gladstone DJ, Rodan LH, Sahlas DJ, et al. A citywide prehospital protocol increases access to stroke thrombolysis in Toronto. Stroke 2009;40(12):3841-3844

7 Lin CB, Peterson ED, Smith EE, et al. Emergency medical service hospital prenotification is associated with improved evaluation and treatment of acute ischemic stroke. Circ Cardiovasc Qual Outcomes 2012;5(04):514-522

8 Fassbender K, Balucani C, Walter S, Levine SR, Haass A, Grotta J. Streamlining of prehospital stroke management: the golden hour. Lancet Neurol 2013;12(06):585-596

9 Fassbender K, Grotta JC, Walter S, Grunwald IQ, RagoschkeSchumm A, Saver JL. Mobile stroke units for prehospital thrombolysis, triage, and beyond: benefits and challenges. Lancet Neurol 2017;16(03):227-237
10 Ebinger M, Winter B, Wendt M, et al; STEMO Consortium. Effect of the use of ambulance-based thrombolysis on time to thrombolysis in acute ischemic stroke: a randomized clinical trial. JAMA 2014;311(16):1622-1631

11 Weber JE, Ebinger M, Rozanski M, et al; STEMO-Consortium. Prehospital thrombolysis in acute stroke: results of the PHANTOM-S pilot study. Neurology 2013;80(02):163-168

12 Kummer BR, Lerario MP, Ganzman AC, et al. Establishing the First Mobile Stroke Unit in New York City. International Stroke Conference; 2/22/2017; Houston, Texas, USA

13 King LHK, Dragan KL, Driver CR, et al. Manhattan Community District 8: Upper East Side. Vol. 8, Issue 59. New York, NY, USA: New York City Department of Health and Mental Hygiene; 2015:1-16

14 Citydata. Carthage, IL: Advameg, Inc. c 2017. Upper East Side (UES) neighborhood in New York, New York (NY), 10021, 10028, 10128 detailed profile. Available at: http://www. city-data.com/neighborhood/Upper-East-Side-New-York-NY. html. Accessed July 15, 2017

15 Bleiwas KB, DiNapoli TP. An Economic Snapshot of Washington Heights and Inwood, Report 2-2016. New York: Office of the New York State Comptroller, New York City Public Information Office. 2015. Available at: https://www.osc.state.ny.us/osdc/rpt2-2016. pdf. Accessed July 15, 2017

16 Fonarow GC, Smith EE, Saver JL, et al. Improving door-to-needle times in acute ischemic stroke: the design and rationale for the American Heart Association/American Stroke Association's Target: stroke initiative. Stroke 2011;42(10):2983-2989 